\title{
Holographic dark energy with the sign-changeable interaction term
}

\author{
M. Abdollahi Zadeh ${ }^{1}$, A. Sheykhi ${ }^{1,2 *}$, H. Moradpour ${ }^{2 \dagger}$ \\ 1 Physics Department and Biruni Observatory, College of Sciences, Shiraz University, Shiraz 71454, Iran \\ ${ }^{2}$ Research Institute for Astronomy and Astrophysics of Maragha (RIAAM), P.O. Box 55134-441, Maragha, Iran
}

\begin{abstract}
We use three IR cutoffs, including the future event horizon, the Hubble and Granda-Oliveros (GO) cutoffs, to construct three holographic models of dark energy. Additionally, we consider a Friedmann-Robertson-Walker (FRW) universe filled by a dark matter (DM) and a dark energy that interact with each other through a mutual sign-changeable interaction. Thereinafter, we address the evolution of the some cosmological parameters, such as the equation of state and dimensionless density parameters of dark energy as well as the deceleration parameter, during the cosmic evolution from the matter dominated era until the late time acceleration. We observe that a holographic dark energy (HDE) model with Hubble cutoff interacting with DM may be in line with the current universe. Our study shows that models with the future event horizon as the IR cutoff or the GO cutoff are in good agreement with the observational data. In fact, we find out that these obtained models can predict the universe transition from a deceleration phase to the acceleration one in a compatible way with observations. The three obtained models may also allow the equation of state parameter to cross the phantom line, a result which depends on the values of the system's constants such as the value of the interaction coupling constant.
\end{abstract}

\section{INTRODUCTION}

Observations data from type Ia supernovae (SNIa) [1] 4 , the Large Scale Structure (LSS) [5] [ ] and the cosmic microwave background (CMB) anisotropies [9 11] obviously signal us an accelerating universe at the present time. From theoretical point of view, this expanding phase of the universe is supported by an unknown source called dark energy (DE) [12]. The simplest model, which consists of a fluid with positive energy density and negative pressure, is called cosmological constant and suffers from some problems such as the fine-tuning and the coincidence problems [12]. Although, many different models, such as quintessence [13, 14], phantom (ghost) field 15, 16], k-essence [17 19], Chaplygin gas 20, 21], agegraphic DE [22 34] and ghost DE models [35 40] have been proposed to justify the current accelerating phase of the universe expansion, $\mathrm{DE}$ is still a confusing topic in modern cosmology [41 43] and its nature and origin is an unknown problem.

Following the developments in quantum theory of gravity, the motivation to solve the DE problem, including its nature and behavior, has arisen a lot of attentions [44]. An example of such effort is the HDE models [45 [51]. The area-entropy relation is the backbone of this hypothesis claiming that black hole relates the short distance cutoff to the long distance cutoff, which leads to an upper bound for the zero-point energy density [45]. Based on this theory, we have $\rho_{D}=3 c^{2} M_{p}^{2} L^{-2}$ for the energy density of HDE, where $c^{2}$ and $L$ are, respectively, a constant and the infrared (IR) cutoff, and $M_{p}$ is the reduced Planck mass [46, 47]. One of the basic assumption in the HDE models is the suitable IR cutoff which leads to an accelerated universe. The simplest choice for the IR cutoff is the Hubble radius which is defined as, $L=H^{-1}$. However, it was argued that Hubble radius cannot lead to an accelerating universe [46], unless the interaction between DE and DM is taken into account [48, 49]. In 2008, Granda and Oliveros (GO) proposed a combination of the Hubble parameter and its first derivative as the IR cutoff of HDE models, namely $L=\left(\alpha H^{2}+\beta \dot{H}\right)^{-1 / 2}$ where $\alpha$ and $\beta$ are constant [52, 53]. This cutoff which is known as the GO cutoff avoids the causality problem and solves the coincidence problem and extensively investigated in the literatures [54, 55].

Moreover, observations indicate that the possibility of a mutual interaction between the DM and DE, which may solve the coincidence problem, is not zero [56]. The simplest form of this mutual interaction can be written as $Q=3 b^{2} H \rho$ in which $b^{2}$ is a constant and $\rho$ can be the energy density of DM, DE or even their sum [56]. Since $b^{2}$ is constant and $H$ and $\rho$ are positive quantities, the sign of the interaction term is not changed during the cosmic evolution. However, recent investigations confirm that the sign of the interaction between DM and DE is changed during the history of the universe and in particular in the redshift $0 \cdot 45 \leq z \leq 0 \cdot 9$ [57]. Clearly, the sign-changeable interaction term cannot be described by the $Q=3 b^{2} H \rho$ expression. Following [57], Wei proposed the sign-changeable interaction term as $Q=q(\alpha \dot{\rho}+3 \beta H \rho)$ for the mutual interaction [58, 59]. In this new expression, both $\alpha$ and $\beta$ are dimensionless constant and $q=-1-\dot{H} / H^{2}$ is the deceleration parameter. The key ingredient is the deceleration parameter $q$ in the interaction $Q$, and hence the interaction $Q$ can change its sign when our universe changes from

\footnotetext{
* asheykhi@shirazu.ac.ir

$\dagger$ h.moradpour@riaam.ac.ir
} 
deceleration phase $(q>0)$ to acceleration $(q<0)$. The $\alpha \dot{\rho}$ and $\beta H \rho$ terms are introduced from the dimensional point of view [58, 60, 61], and one can ignore the $\alpha \dot{\rho}$ term by considering $\alpha=0$ [58, 60, 61]. It is worth noting that the $Q(\rho, \dot{\rho})$ form has previously been introduced to describe the mutual interaction between the dark sectors of cosmos [60, 61]. Now, bearing the Firedmann equation in mind $\left(H^{2} \propto \rho\right)$, we find out that the $Q(\rho, \dot{\rho})$ interaction can indeed be written in the $Q(H, \dot{H})$ form. On the other hand, since $q=q(H, \dot{H})$, the $Q=q(\alpha \dot{\rho}+3 \beta H \rho)$ interaction can also be written in the form $Q(H, \dot{H})$, meaning that in the Wei's approach, the appearance of the deceleration parameter, $q$, in the interaction term $Q$ is quite acceptable [58, 59].

In the present work, we are interested in studying the cosmological consequences of considering a sign-changeable interaction term in HDE model. We shall take $Q=3 b^{2} H q \rho$, where $\rho=\rho_{m}+\rho_{D}$ is the total energy density. We will consider three cutoffs, including the future event horizon, the Hubble and GO cutoffs, to build the energy density of HDE which helps us in providing three models for DE. Thereinafter, we investigate the evolution of the system parameters, such as the state parameter as well as the deceleration and dimensionless density parameters, during the cosmos evolution from the matter dominated era to the current accelerating epoch.

The paper is organized as follows. In section II, we use the future event horizon as the IR cutoff to build the HDE, and study the evolution of the system's cosmological parameters. Sections III and IV also include the HDE models with the Hubble and GO cutoffs, respectively. To have a better understanding of the system evolution, we plot the cosmological parameters, such as $q$, the equation of state and dimensionless density parameters, in terms of the redshift $z$. We finish with summary and concluding remarks in the last section.

\section{HDE WITH THE SIGN-CHANGEABLE INTERACTION WITH FUTURE HORIZON AS IR CUTOFF}

We consider a homogeneous and isotropic flat FRW universe which is described by the line element

$$
d s^{2}=-d t^{2}+a^{2}(t)\left[d r^{2}+r^{2} d \Omega^{2}\right],
$$

where $a(t)$ is scale factor. The first Friedmann equation is written as

$$
H^{2}=\frac{\rho}{3 M_{p}^{2}},
$$

where $\rho$ is the total energy density, which satisfy the conservation law

$$
\dot{\rho}+3 H(\rho+p)=0 .
$$

We assume the two dark sectors of the universe, namely pressureless DM and HDE exchange energy and thus they no longer satisfy the conservation equations, separately. Instead, they satisfy the semi-conservation equations as

$$
\begin{aligned}
& \dot{\rho}_{m}+3 H \rho_{m}=Q, \\
& \dot{\rho}_{D}+3 H\left(1+\omega_{D}\right) \rho_{D}=-Q .
\end{aligned}
$$

where $\omega_{D} \equiv p_{D} / \rho_{D}$ is the equation of state parameter of HDE, and $Q$ denotes the interaction term between DE and DM. As it is obvious, DE (DM) decays into DM (DE) for $Q>0(Q<0)$. Following [58, 59], we assume the interaction term has the following form

$$
Q=3 b^{2} H q\left(\rho_{D}+\rho_{m}\right),
$$

where $b^{2}$ is the coupling constant and $q$ is the deceleration parameter defined as

$$
q=-\frac{\ddot{a} a}{\dot{a}^{2}}=-1-\frac{\dot{H}}{H^{2}} .
$$

From Eq. (6), it is obvious that the transition of the universe from the deceleration $(q>0)$ to an acceleration $(q<0)$ phase, changes the sign of the interaction term $Q$. Defining, as usual, the dimensionless density parameters as

$$
\Omega_{m}=\frac{\rho_{m}}{3 M_{p}^{2} H^{2}}, \quad \Omega_{D}=\frac{\rho_{D}}{3 M_{p}^{2} H^{2}},
$$

we can rewrite the first Friedmann equation in the form

$$
\Omega_{m}+\Omega_{D}=1 .
$$


Also, if we take the time derivative of the ratio of the energy densities, $r=\rho_{m} / \rho_{D}$, we find

$$
\dot{r}=3 b^{2} H q+6 b^{2} H q r+3 b^{2} H q r^{2}+3 H r \omega_{D},
$$

where we have used Eqs. (4) and (5). Solving for $w_{D}$, we arrive at

$$
\omega_{D}=\frac{\dot{r}-3 b^{2} H q-6 b^{2} H q r-3 b^{2} H q r^{2}}{3 H r} .
$$

Using Eqs. (8), (9), one obtains

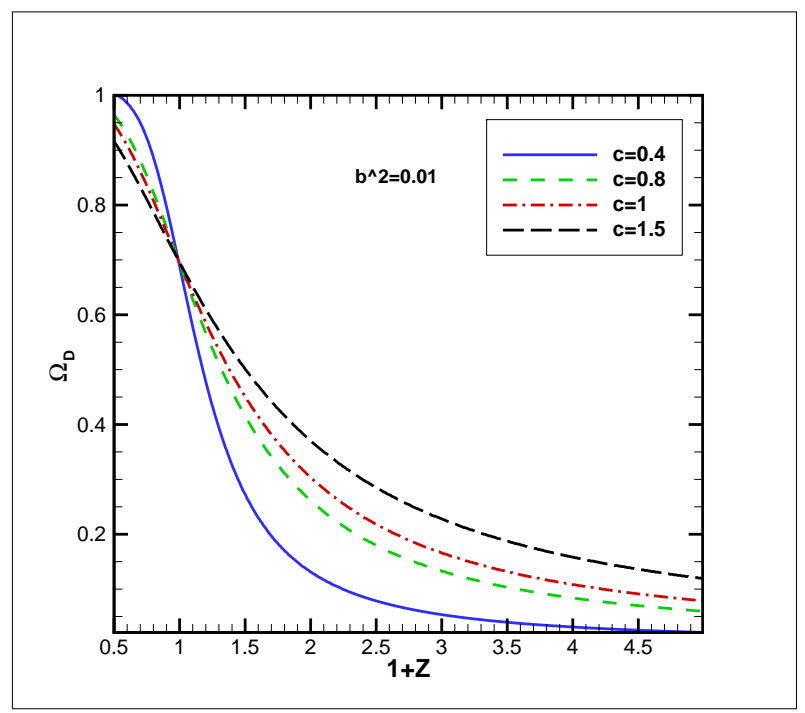

FIG. 1: Evolution of $\Omega_{D}$ versus redshift parameter $z$ for HDE with Future cutoff for a fixed interaction parameter with coupling interaction $b^{2}$ but for different values of the constant $c$.

$$
r=\frac{\Omega_{m}}{\Omega_{D}}=\frac{1-\Omega_{D}}{\Omega_{D}}
$$

which implies

$$
\dot{r}=-\frac{\dot{\Omega}_{D}}{\Omega_{D}^{2}}
$$

Taking into account the fact that $\dot{\Omega}_{D}=\Omega_{D}^{\prime} H$, after using Eq. (13), we can rewrite Eq. (11) as

$$
\omega_{D}=\frac{-\Omega_{D}^{\prime} / \Omega_{D}^{2}-3 b^{2} q-6 b^{2} q r-3 b^{2} q r^{2}}{3 r},
$$

where the dot and the prime stand for the derivative with respect to the time and $x=\ln a$, respectively. Considering the Hubble radius as the IR cutoff, it is easy to show that $r$ is a constant [48, 50]. Here, we use the future event horizon as the IR cutoff and so $r$ is no longer a constant [46]. The future event horizon is defined as [46, 62]

$$
R_{h}=a(t) \int_{t}^{\infty} \frac{d t}{a(t)}=\frac{c \sqrt{1+r}}{H}
$$

where $c$ is a constant, and it leads to

$$
\dot{R_{h}}=H R_{h}-1=c \sqrt{1+r}-1 .
$$

Taking the time derivative of Eq. (2), after using Eqs. (44), (5) and (8), we obtain

$$
\frac{\dot{H}}{H^{2}}=-\frac{3}{2} \Omega_{D}\left(1+r+\omega_{D}\right)
$$


Moreover, using (16) and (17), we obtain

$$
\omega_{D}=-\frac{1}{3}-\frac{2 \sqrt{\Omega_{D}}}{3 c}-\frac{b^{2} q}{\Omega_{D}}
$$

Combining Eqs. (16) and (17) we get,

$$
\frac{\Omega_{D}^{\prime}}{\Omega_{D}^{2}}=\left(1-\Omega_{D}\right)\left(\frac{1}{\Omega_{D}}+\frac{2}{c \sqrt{\Omega_{D}}}-\frac{3 b^{2} q}{\Omega_{D}\left(1-\Omega_{D}\right)}\right) .
$$

Substituting Eq. (17) into (7), one finds

$$
q=\frac{1-\Omega_{D}-\frac{2 \Omega_{D}^{\frac{3}{2}}}{c}}{2+3 b^{2}} .
$$

Combining this equation with Eq. (18), we find out that, for the non-interacting case $\left(b^{2}=0\right)$, we have $\omega_{D}=-1$ in late time where $\Omega_{D} \rightarrow 1$ provided we take $c=1$. In addition, it is easy to check that, for $b^{2}=0$ and $c=1$, we have $q=-1$ provided $\Omega_{D} \rightarrow 1$.
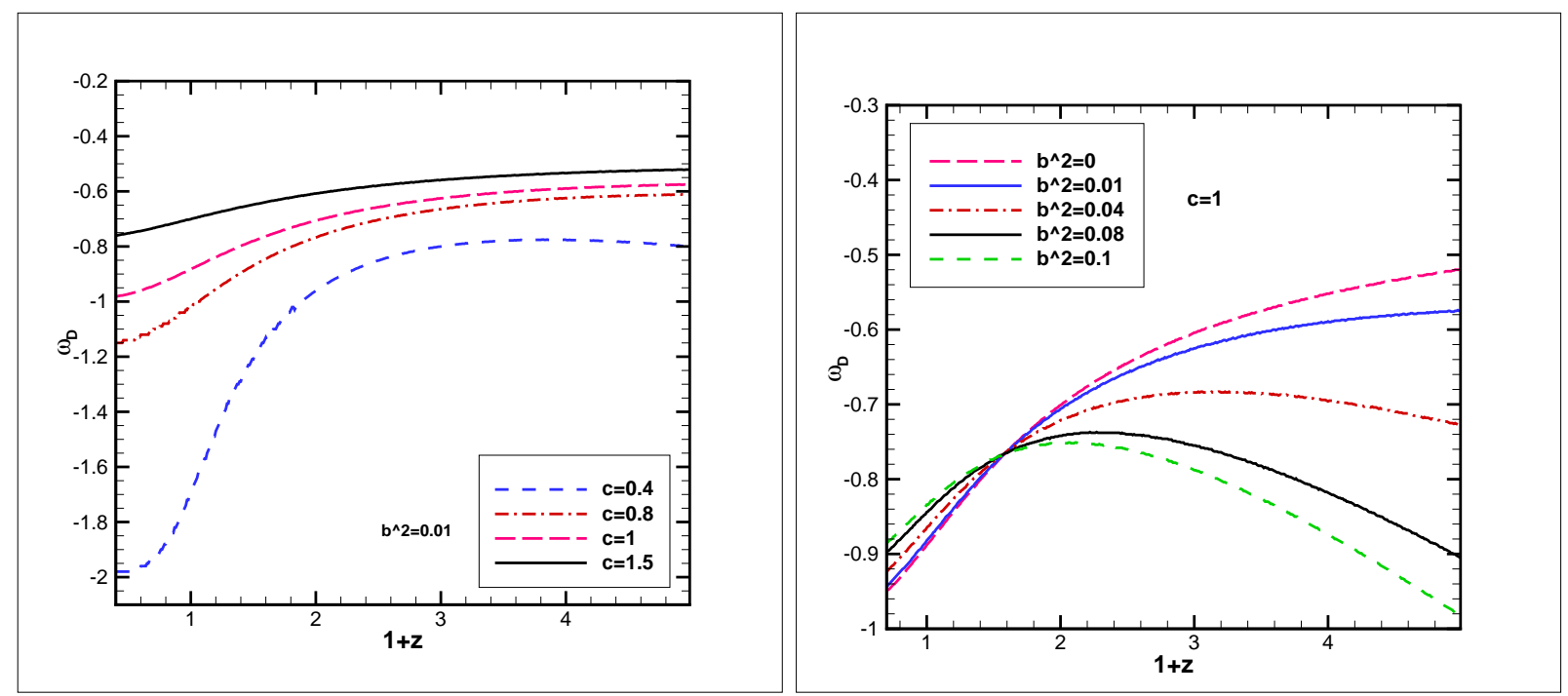

FIG. 2: The evolution of $\omega_{D}$ versus redshift parameter $z$ for HDE with Future cutoff. Left panel corresponds to a fixed couplings between DE and DM and the right panel shows the case in which $c$ is constant.

The evolution of $\Omega_{D}$ in terms of redshift parameter $(1+z)$ with respect to the constant $b^{2}$ is plotted in Fig. 1 . From this figure we see that, in the early universe where $z \rightarrow \infty$ we have $\Omega_{D} \rightarrow 0$, while at the late time where $1+z \rightarrow 0$ we have $\Omega_{D} \rightarrow 1$. It is also apparent that, at fixed $b^{2}$, the DE role in the early universe will be highlighted with increasing the value of $c$. Additionally, in the long run limit $(1+z \rightarrow 0)$, DE plays a more effective role with decreasing the value of $c$. The equation of state (EoS) parameter given in (18) is also plotted in Fig. 2 showing that, for a fixed $b^{2}$, the EoS parameter can cross the phantom line for $c<1$, and when $c>1$, we always have $\omega_{D}>-1$.

The behaviour of the deceleration parameter $q$ is plotted in Fig. 3 showing that there is a deceleration expansion at the early time followed by an acceleration expansion. In addition, the transition from the deceleration phase to the acceleration one happen at $z \approx 0.6$ which is consistent with recent observations 63 65]. As Fig. 3 shows, for fixed $b^{2}$, the universe expansion may experience a phantom phase at current time while $c>1$. Moreover, for two universes with the same $b^{2}$ and different values of $c$, the universe with larger amount of $c$ gets its phase transition point earlier than the other universe. Finally, for fixed $c$ and for the current stage of the universe, this model predicts that the universe expansion phase may fall in the phantom era as a function of $b^{2}$.

\section{HDE WITH THE SIGN-CHANGEABLE INTERACTION WITH HUBBLE CUTOFF}

In this section, we use the Hubble radius $\left(L=H^{-1}\right)$ as IR cutoff to study the effects of a sign-changeable interaction in the HDE model. In this case we have $\rho_{D}=3 c^{2} M_{p}^{2} H^{2}$, where $c$ is a constant. Finally, bearing the $\Omega_{D}=$ 

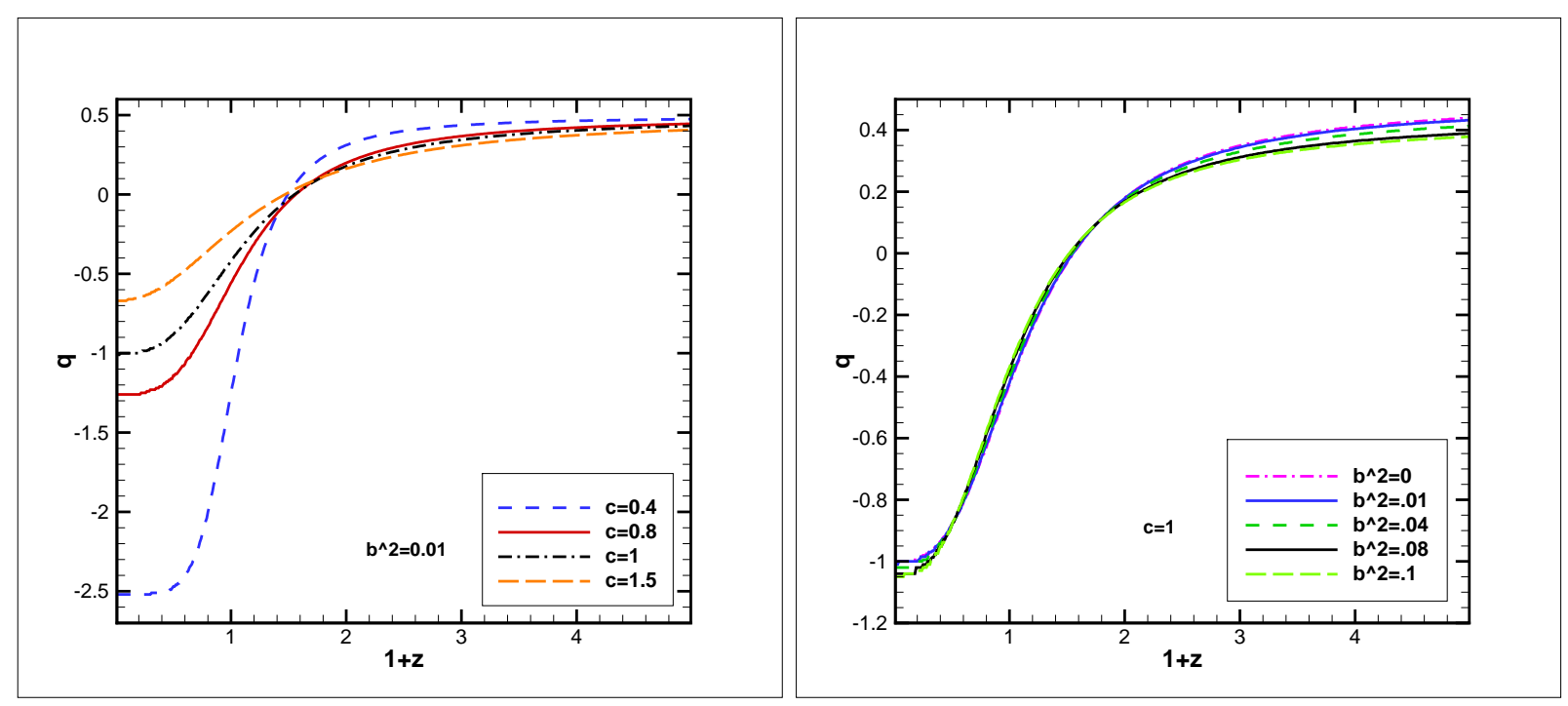

FIG. 3: The evolution of the deceleration parameter $q$ against redshift parameter $z$ for HDE with Future cutoff. Left panel corresponds to a fixed couplings between DE and DM and the right panel shows the case $c$ is constant.
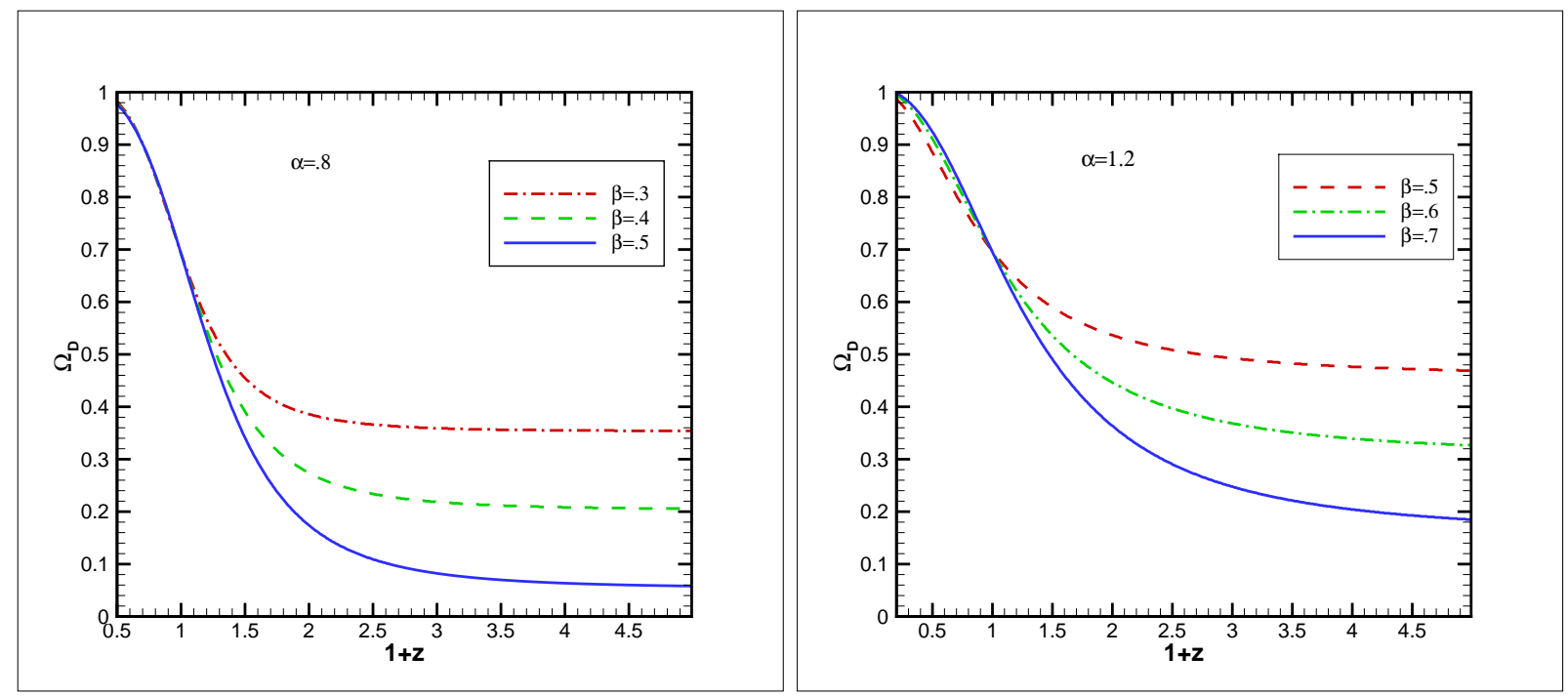

FIG. 4: The evolution of $\Omega_{D}$ versus the $1+z$ parameter for HDE with GO cutoff and $b^{2}=0 \cdot 01$. Left panel corresponds to $\alpha<1$ and the right panel shows the $\alpha>1$ case.

$\rho_{D} /\left(3 M_{p}^{2} H^{2}\right)$ relation in mind, we find out $\Omega_{D}=c^{2}$ meaning that $c^{2}$ should meet the $0 \leq c^{2} \leq 1$ condition [66]. It is worth mentioning that, in the non-interacting model we arrive at $\omega_{D}=0$ for the HDE with Hubble radius as the IR cutoff [46]. This implies that in this case the accelerated expansion of the universe cannot be achieved [46]. It is also a matter of calculation to combine the $\rho_{D}=3 c^{2} M_{p}^{2} H^{2}$ expression with Eqs. (5) and (17), and using the $Q=3 b^{2} H q \rho=3 b^{2} H q\left(\rho_{D}+\rho_{m}\right)$ relation for the mutual interaction to get

$$
\omega_{D}=-\frac{b^{2} q}{c^{2}\left(1-c^{2}\right)}
$$

where

$$
q=-1-\frac{\dot{H}}{H^{2}}=\frac{1-c^{2}}{2\left(1-c^{2}\right)+3 b^{2}}
$$


It is obvious that in the absence of the interaction term, where $b^{2}=0$, we get $w_{D}=0$ and $q=1 / 2>0$ which refer to a decelerated universe. Now, inserting Eq. (22) into Eq. (21), we obtain

$$
\omega_{D}=-\frac{b^{2}}{c^{2}\left[2\left(1-c^{2}\right)+3 b^{2}\right]} .
$$

Clearly, for $2\left(1-c^{2}\right)+3 b^{2}>0$, we have $\omega_{D}<0$.
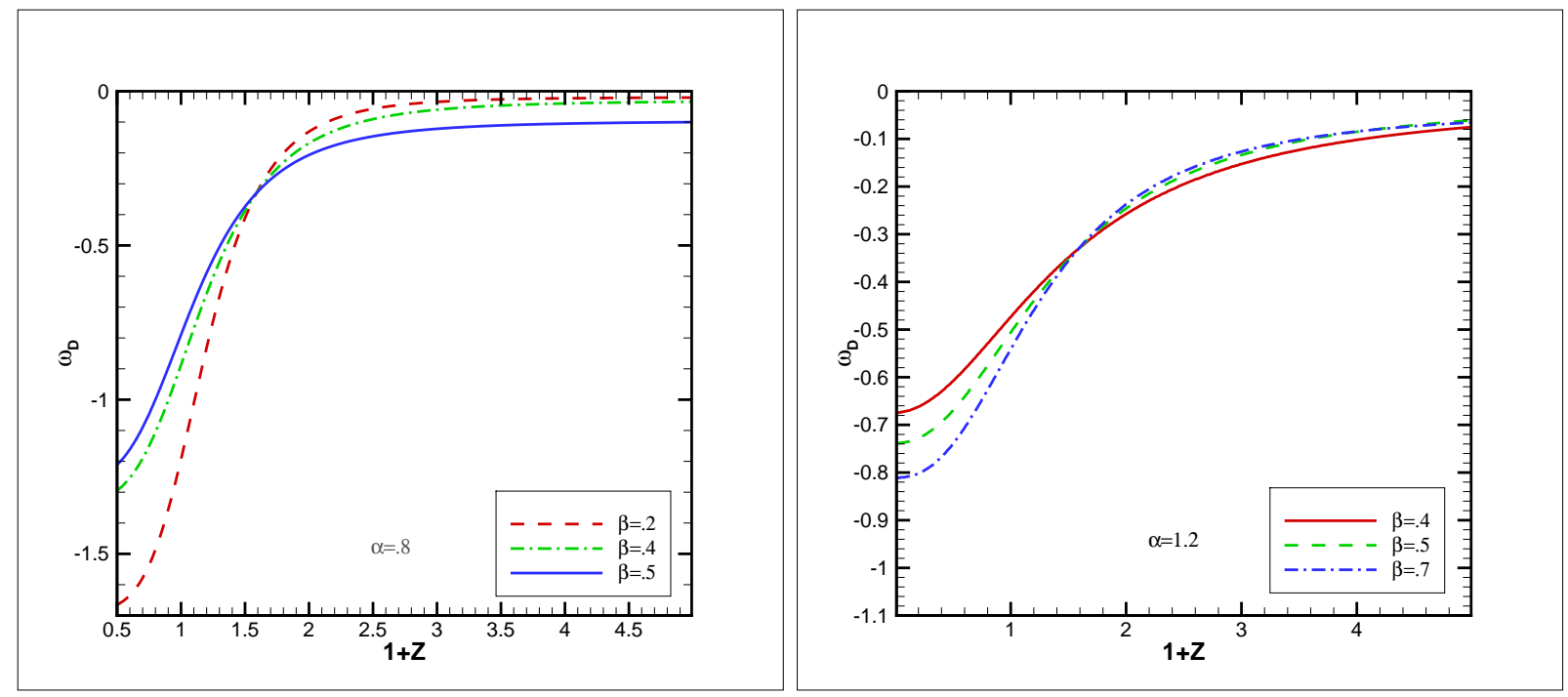

FIG. 5: The evolution of $\omega_{D}$ versus the $1+z$ parameter for HDE with GO cutoff and $b^{2}=0 \cdot 01$. Left panel corresponds to $\alpha<1$ and the right panel shows the $\alpha>1$ case.

\section{HDE WITH THE SIGN-CHANGEABLE INTERACTION WITH GO CUTOFF}

The third cutoff, introduced by Granda and Oliveros (GO) [52], is the generalization of the Ricci scalar [67] in which we have $L=\left(\beta \dot{H}+\alpha H^{2}\right)^{-1 / 2}$ where $\alpha$ and $\beta$ are constant, and therefore

$$
\rho_{D}=3 M_{p}^{2}\left(\alpha H^{2}+\beta \dot{H}\right),
$$

which can also be rewritten

$$
\frac{\dot{H}}{H^{2}}=\frac{\Omega_{D}-\alpha}{\beta} .
$$

Taking the time derivative of Eq. (2) and using Eqs. (4), (5), (8) and (9), we reach

$$
\frac{\dot{\rho}_{D}}{3 M_{p}^{2} H^{3}}=\frac{2 \dot{H}}{H^{2}}+3\left(1-\Omega_{D}\right)-3 b^{2} q .
$$

From Eq. (8), we obtain

$$
\dot{\Omega}_{D}=\frac{\dot{\rho}_{D}}{3 M_{p}^{2} H^{2}}-2 \Omega_{D} \frac{\dot{H}}{H} .
$$

Combining Eqs. (25) and (26) with (27) to get

$$
\Omega_{D}^{\prime}=\left(1-\Omega_{D}\right)\left[3+\frac{2}{\beta}\left(\Omega_{D}-\alpha\right)\right]-3 b^{2} q .
$$


Inserting Eqs. (24), (25) and (26) into (15), and combining Eqs. (25) and (7), we find the EoS and deceleration parameter as

$$
\begin{gathered}
\omega_{D}=-\frac{1}{\Omega_{D}}\left[1+\frac{2}{3}\left(\frac{\Omega_{D}-\alpha}{\beta}\right)\right], \\
q=-1-\frac{\dot{H}}{H^{2}}=-1-\frac{\Omega_{D}-\alpha}{\beta} .
\end{gathered}
$$

The evolution of HDE density parameter $\left(\Omega_{D}\right)$ in terms of redshift parameter $(1+z)$, whenever $b^{2}=0 \cdot 01$, is shown in Fig. 4. As it is obvious from Fig. 4, we have $\Omega_{D} \rightarrow 0$ and $\Omega_{D} \rightarrow 1$ in the early universe $(1+z \rightarrow \infty)$ and the $1+z \rightarrow 0$ limit, respectively.

Since Eq. (29) indicates that we have $\omega_{D}=-1$ while $\Omega_{D} \rightarrow 1$ and $\alpha=1$ simultaneously, we plot the $\alpha<1$ and $\alpha>1$ cases separately in Fig. [5. As it is obvious from this figure, the model can show a phantom-like behavior $\left(\omega_{D}<-1\right)$ for $\alpha<1$. Additionally, for the $\alpha>1$ case, $\omega_{D}$ is always larger than -1 . The behavior of $q$ is also shown
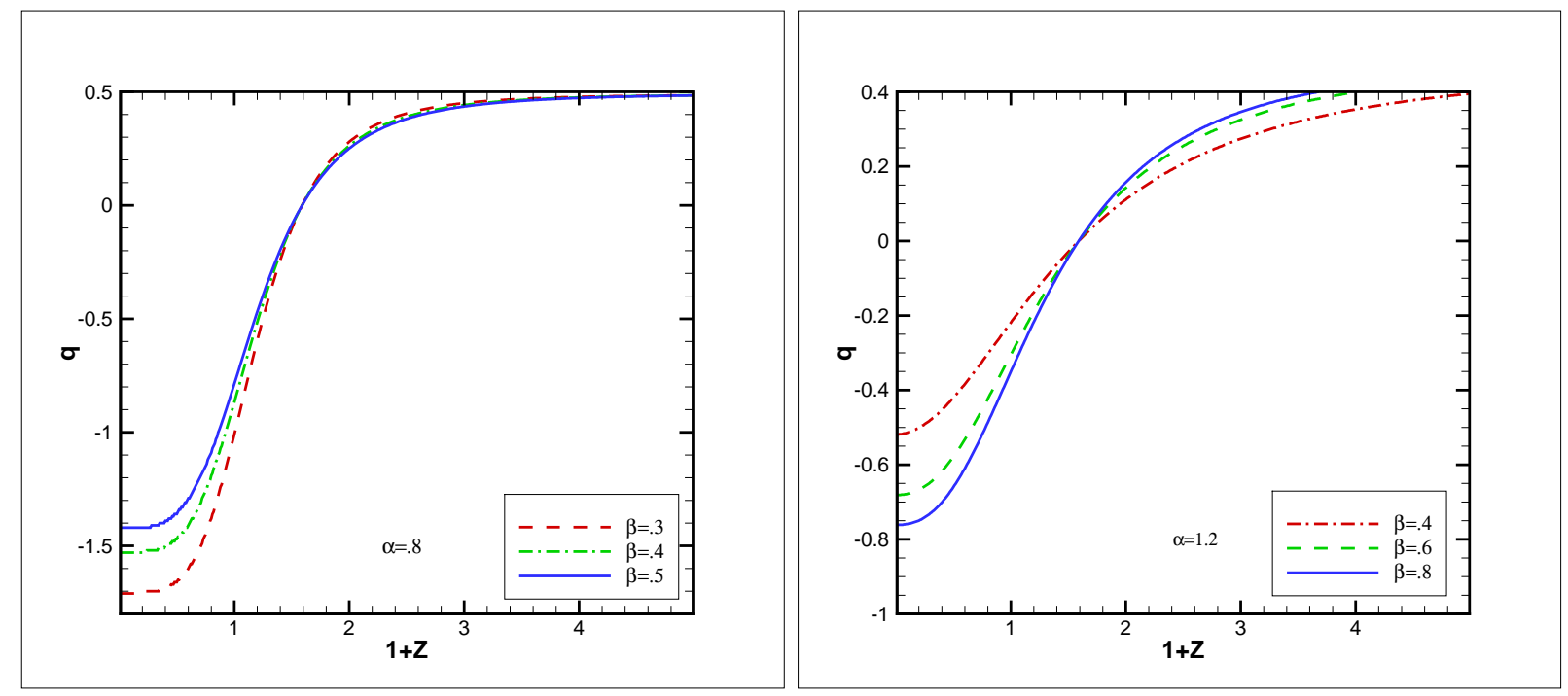

FIG. 6: The evolution of $q$ versus the $1+z$ parameter for HDE with GO cutoff and $b^{2}=0 \cdot 01$. Left panel corresponds to $\alpha<1$ and the right panel shows the case $\alpha>1$.

in Fig. 6. It is obvious that the universe experiences a transition from a deceleration phase to an acceleration one around $z \approx 0 \cdot 6$, compatible with observation [63]. It is also useful to note that Eq. (30) shows $q \rightarrow-1$ while $\Omega_{D} \rightarrow 1$ and $\alpha=1$.

\section{SUMMARY AND CONCLUDING REMARKS}

Throughout this paper, we considered a universe filled by dark matter and dark energy, in which there is a signchangeable interaction $\left[Q=3 b^{2} H q\left(\rho_{D}+\rho_{m}\right)\right]$ between the dark sectors. Considering the future event horizon, the Hubble radius and GO as the system IR cutoffs, we followed the HDE idea to obtain three models for dark energy.

At first, we used the future event horizon as the IR cutoff to construct the HDE density profile. In addition, we studied the evolution of density parameter, the deceleration parameter and the EOS parameter. Our study shows that the model is compatible with some recent observations, and it predicts a transition from the deceleration phase to an acceleration one at around $z \approx 0 \cdot 6$. Our check indicates that, for fixed $b^{2}$, we have $\omega_{D}>-1$ for $c>1$. We also saw that, for fixed $b^{2}$, the phantom-like behavior $\left(\omega_{D}<-1\right)$ may be obtained for $c<1$.

Moreover, we focused on the Hubble and the GO cutoffs and found out that these cutoffs may also lead to HDE models compatible with observations. In fact, we saw that, unlike the non-interacting HDE model with the Hubble cutoff [46], it is possible to have $\omega_{D}<0$ and $q<0$ in the interacting case. In addition, our study shows that the model with the GO cutoff can predict the universe transition from a deceleration phase to an acceleration one at around $z \approx 0 \cdot 6$. We finally found out that the EOS of the model with GO cutoff may also cross the phantom line. 


\section{Acknowledgments}

We also thank Shiraz University Research Council. This work has been supported financially by Research Institute for Astronomy \& Astrophysics of Maragha (RIAAM), Iran.

[1] A. G. Riess et al., Observational evidence from supernovae for an accelerating Universe and a cosmological constant, Astron. J. 1161009 (1998) arXiv:astro-ph/9805201.

[2] S. Perlmutter et al., Measurements of omega and lambda from 42 high-redshift supernovae, Astrophys. J. 517565 (1999) arXiv:astro-ph/9812133.

[3] P. deBernardis, et al., A flat Universe from high-resolution maps of the cosmic microwave background radiation, Nature 404955 (2000) arXiv:astro-ph/0004404v1].

[4] S. Perlmutter, et al., New Constraints on M, , and $w$ from an Independent Set of 11 High-Redshift Supernovae Observed with the Hubble Space Telescope,Astrophys. J. 598102 (2003) arXiv:astro-ph/0309368v1].

[5] M. Colless et al., The 2dF Galaxy Redshift Survey: Spectra and redshifts, Mon. Not. R. Astron. Soc. 328, 1039 (2001) arXiv:astro-ph/0106498v3].

[6] M. Tegmark etal., Cosmological parameters from SDSS and WMAP, Phys. Rev. D 69, 103501 (2004)[ arXiv:astro-ph/0310723 v2].

[7] S. Cole et al., The 2dF Galaxy Redshift Survey:Power-spectrum analysis of the final dataset and cosmological implications, Mon. Not. R. Astron. Soc. 362, 505 (2005) arXiv:astro-ph/0501174v2].

[8] V. Springel, C. S. Frenk, and S. M. D. White, The large-scale structure of the Universe, Nature(London) 440, 1137 (2006) arXiv:astro-ph/0604561.

[9] S. Hanany et al., MAXIMA-1:A Measurement of the Cosmic Microwave Background Anisotropy on Angular Scales of 10'-5, Astrophys. J. Lett. 545, L5 (2000) arXiv:astro-ph/0005123v3].

[10] C. B. Netterfield et al., A Measurement by BOOMERANG of Multiple Peaks in theAngular Power Spectrum of the Cosmic Microwave Background, Astrophys. J. 571, 604 (2002) arXiv:astro-ph/0104460v3].

[11] D. N. Spergel et al., First Year Wilkinson Microwave Anisotropy Probe (WMAP) Observations: Determination of Cosmological Parameters, Astrophys. J. Suppl. 148, 175 (2003)[ arXiv:astro-ph/0302209v3].

[12] M. Roos, Introduction to Cosmology (John Wiley and Sons, UK, 2003)

[13] C. Wetterich, Cosmology And The Fate Of Dilatation Symmetry, Nucl. Phys. B302, 668 (1988).

[14] B. Ratra and J. Peebles, Cosmological Consequences Of A Rolling Homogeneous Scalar Field, Phys. Rev. D 37, 3406 (1988).

[15] R. R. Caldwell, A Phantom Menace?, Phys. Lett. B 545, 23 (2002) astro-ph/9908168].

[16] R. R. Caldwell, M. Kamionkowski and N. N. Weinberg, Phantom Energy and Cosmic Doomsday,Phys. Rev. Lett.91, 071301 (2003) astro-ph/0302506.

[17] T. Chiba, T. Okabe, and M. Yamaguchi, Kinetically driven quintessence, Phys. Rev. D 62, 023511 (2000) arXiv:astro-ph/9912463.

[18] C. Armendariz-Picon, V. F. Mukhanov and P. J. Steinhardt, A dynamical solution to the problem of a small cosmological constant and late-time cosmic acceleration, Phys. Rev. Lett. 85, 4438 (2000) arXiv:astro-ph/0004134.

[19] C. Armendariz-Picon, V. F. Mukhanov and P. J. Steinhardt, Essentials of k-essence, Phys. Rev.D63, 103510 (2001) arXiv:astro-ph/0006373.

[20] A. Y. Kamenshchik, U. Moschella and V. Pasquier, An alternative to quintessence, Phys. Lett. B511, 265 (2001) arXiv:gr-qc/0103004.

[21] M. C. Bento, O. Bertolami, and A. A. Sen, Generalized Chaplygin Gas, Accelerated Expansion and Dark Energy-Matter Unification, Phys. Rev. D 66, 043507 (2002) arXiv:gr-qc/0202064.

[22] R. G. Cai, A Dark Energy Model Characterized by the Age of the Universe, Phys. Lett. B 657, 228 (2007) arXiv:0707.4049 [hep-th]].

[23] H. Wei and R. G. Cai, A new model of agegraphic dark energy ,Phys. Lett. B 660, 113 (2008) arXiv:0708.0884 [astro-ph]].

[24] K. Y. Kim, H.W. Lee, and Y. S. Myung, Instability of agegraphic dark energy models, Phys. Lett. B 660, 118 (2008) arXiv:0709.2743 gr-qc]].

[25] H. Wei and R. G. Cai, Cosmological constraints on new agegraphic dark energy, Phys. Lett. B663, 1 (2008) arXiv:0708.1894 [astro-ph]].

[26] J. P. Wu, D. Z. Ma, and Y. Ling, Quintessence reconstruction of the new agegraphic dark energy model, Phys. Lett. B663, 152 (2008) arXiv:0805.0546 [hep-th]].

[27] J. Zhang, X. Zhang, and H. Liu,Agegraphic dark energy as a quintessence, Eur. Phys. J. C 54, 303 (2008) arXiv:0801.2809 [astro-ph]].

[28] H. Wei and R. G. Cai, Interacting agegraphic dark energy, Eur. Phys. J. C 59, 99 (2009) arXiv:0707.4052 [hep-th]]; H. Wei and R. G. Cai, Statefinder Diagnostic and w-w' Analysis for the Agegraphic Dark Energy Models without and with Interaction, Phys. Lett. B 655, 1 (2007), arXiv:0707.4526;

H. Wei and R. G. Cai, Interacting Agegraphic Dark Energy, Eur. Phys. J. C 59, 99 (2009), arXiv:0707.4052

[29] I. P. Neupane, A note on agegraphic dark energy, Phys. Lett. B673, 111 (2009) arXiv:0708.2910 [hep-th]]. 
[30] A. Sheykhi, Interacting agegraphic dark energy models in non-flat universe, Phys. Lett. B 680, 113 (2009) arXiv:0907.5144 [hep-th]].

[31] A. Sheykhi Interacting agegraphic tachyon model of dark energy, 682, 329 (2010) arXiv:0907.2491] [hep-th]].

[32] A. Sheykhi Interacting new agegraphic dark energy in non-flat Brans-Dicke cosmology, Phys. Rev. D 81, 023525 (2010) arXiv:0908.0606 [gr-qc]].

[33] A. Sheykhi and M. Jamil,Interacting HDE and NADE in Brans-Dicke Chameleon Cosmology, Phys. Lett. B694, 284 (2011) arXiv:1010.0385 [hep-th]].

[34] A. Sheykhi and M. R. Setare,Interacting new agegraphic viscous dark energy with varying G, Int. J. Theor. Phys.49, 2777 (2010) arXiv:1003.1109 [physics.gen-ph]].

[35] N. Ohta, Dark energy and QCD ghost, Phys. Lett. B 695, 41 (2011), arXiv:1010.1339.

[36] R. G. Cai, Q. Su, Z. L. Tuo, H. B. Zhang, Notes on Ghost Dark Energy, Phys. Rev. D 84,123501 (2011), arXiv:1011.3212.

[37] A. Sheykhi, M. Sadegh Movahed, Interacting ghost dark energy in non-flat universe Gen. Relativ. Gravit. 44,449 (2012), arXiv:1104.4713.

[38] E. Ebrahimi, A. Sheykhi, Instability of QCD ghost dark energy model, Int. J. Mod. Phys. D 20, 2369 (2011), arXiv:1106.3504.

[39] A. Sheykhi, M. Sadegh Movahed, E. Ebrahimi, Tachyon Reconstruction of Ghost Dark Energy, Astrophys. Space Sci. 339, 93 (2012), arXiv:1105.0325.

[40] A. Sheykhi, M. Tavayef, Ghost Dark Energy in a Cyclic Universe, Iran. J. Sci. Technol. Trans. Sci. DOI: 10.1007/s40995016-0083-y.

[41] S. Nojiri and S. D. Odintsov, The new form of the equation of state for dark energy fluid and accelerating universe, Phys. Lett. B 639, 144 (2006), arXiv:hep-th/0606025.

[42] K. Bamba, S. Capozziello, S. Nojiri and S. D. Odintsov, Dark energy cosmology: the equivalent description via different theoretical models and cosmography tests, Astrophys. Space Sci. 342, 155 (2012), arXiv:1205.3421.

[43] M. Li. X. D. Li, S. Wang and and Y. Wang, THE UNIVERSE. 1, 4 (2013).

[44] E. Witten, The cosmological constant from the viewpoint of string theory, [ arXiv:hep-ph/0002297].

[45] A. G. Cohen, D. B. Kaplan and A. E. Nelson, Effective field theory, black holes, and the cosmological constant, Phys. Rev. Lett. 82, 4971 (1999) arXiv:hep-th/9803132

[46] M. Li, A model of holographic dark energy, Phys. Lett. B 603, 1 (2004) arXiv:hep-th/0403127.

[47] S. D. H. Hsu, Entropy bounds and dark energy, Phys. Lett. B 594, 13 (2004) arXiv:hep-th/0403052

[48] D. Pavon, W. Zimdahl,Holographic dark energy and cosmic coincidence, Phys. Lett. B 628, 206 (2005) arXiv:gr-qc/0505020.

[49] A. Sheykhi, Thermodynamics of interacting holographic dark energy with the apparent horizon as an IR cutoff, Classical Quantum Gravity 27, 025007 (2010). arXiv:0910.0510 [hep-th]]

[50] A. Sheykhi,Holographic scalar field models of dark energy, Phys. Rev. D 84, 107302 (2011) arXiv:1106.5697 [arXiv:physics.gen-ph]

[51] H. Wei, Pilgrim Dark Energy, Class. Quant. Grav. 29, 175008 (2012) arXiv:1204.4032;

H. Wei, Modified Holographic Dark Energy, Nucl. Phys. B 819, 210 (2009) arXiv:0902.2030.

[52] L. N. Granda, A. Oliveros, Infrared cut-off proposal for the holographic density, Phys. Lett. B 669 (2008) [arXiv:0810.3149v1 $[\mathrm{gr}-\mathrm{qc}]]$

[53] L. N. Granda, A. Oliveros, New infrared cut-off for the holographic scalar fields models of dark energy, Phys. Lett. B 671275 (2009) 199 arXiv:0810.3663v2 [gr-qc]]

[54] M. Jamil, K. karami, A. Sheykhi, E. Kazemi, Z. Azarmi, Holographic dark energy in Brans-Dicke cosmology with GrandaOliveros cut-off, Int. J. Theor. Phys. 51, 604 (2012).

[55] S. Ghaffari, M. H. Dehghani and A. Sheykhi, Holographic dark energy in the DGP braneworld with GO cutoff, Phys. Rev. D 89(2014) 123009 arXiv:1506.01676];

A. Khodam-Mohammadi, E. Karimkhani, A. Sheykhi, Best values of parameters for interacting HDE with GO IR-cutoff in Brans-Dicke cosmology, Int. J. Mod. Phys. D, 23, 14500813 (2014) arXiv:1409.3115.

[56] B. Wang, E. Abdalla, F. Atrio-Barandela, D. Pavon, Dark Matter and Dark Energy Interactions: Theoretical Challenges, Cosmological Implications and Observational Signatures arXiv:1603.08299 [gr-qc]]

[57] R. G. Cai and Q. P. Su, On the Dark Sector Interactions, Phys. Rev. D 81, 103514 (2010) arXiv:0912.1943v2 [astro-ph.CO]].

[58] H. Wei, Cosmological Evolution of Quintessence and Phantom with a New Type of Interaction in Dark Sector, Nucl. Phys. B 845,381 (2011) arXiv:1008.4968v3]

[59] H. Wei, Cosmological Constraints on the Sign-Changeable Interactions, Commun. Theor. Phys.56, 972 (2011) arXiv:1010.1074 [gr-qc]]

[60] L. P. Chimento, Linear and nonlinear interactions in the dark sector, Phys. Rev. D 81, 043525 (2010) arXiv:0911.5687.

[61] L. P. Chimento, M. Forte and G. M. Kremer, Cosmological model with interactions in the dark sector, Gen. Rel. Grav. 41, 1125 (2009) arXiv:0711.2646.

[62] Q.G. Huang, M. Li, The holographic dark energy in a non-flat universe ,JCAP 0408013 (2004) . arXiv:astro-ph/0404229.

[63] R.A. Daly et al., Improved Constraints on the Acceleration History of the Universe and the Properties of the Dark Energy, Astrophys. J. 677, 1 (2008).

[64] E. Komatsu et al. [WMAP Collaboration], SEVEN-YEAR WILKINSON MICROWAVE ANISOTROPY PROBE (WMAP *) OBSERVATIONS: COSMOLOGICAL INTERPRETATION, Astrophys. J. Suppl. 192, 18 (2011).

[65] V. Salvatelli, A. Marchini, L. L. Honorez and O. Mena, New constraints on coupled dark energy from the Planck satellite experiment, Phys. Rev. D 88, 023531 (2013). 
[66] S. Ghaffari, A. Sheykhi and M. H. Dehghani, Statefinder diagnosis for holographic dark energy in the DGP braneworld, Phys. Rev. D 912, 023007 (2015) arXiv:1504.04008 [gr-qc]].

[67] C. J. Gao, X. L. Chen and Y. G. Shen, A Holographic Dark Energy Model from Ricci Scalar Curvature ,Phys. Rev. D 79, 043511 (2009)[ arXiv:0712.1394 4 [astro-ph]]. 